\title{
TECHNICAL CONDITION OF VEHICLE CHASSIS PARTS AND TYRE
} WEAR

\author{
Liu Xi' ${ }^{1}$, Tang Wen $\mathrm{Ke}^{2}$ \\ ${ }^{1}$ School of Mechatronics \& Automotive Engineering, Chongqing Jiao-tong University, Chongqing, China \\ ${ }^{2}$ School of Mechatronics \& Automotive Engineering, Chongqing Jiao-tong University, Chongqing, China
}

\begin{abstract}
The modern automobile is contains the machinery, electron, communication and computer intelligence as one high-tech product, the multiplication of system has determined its faults diversity, multiple and complex, but regarding the major function of vehicles manned transportation, maintains the normal speed most basic request, the length of automobile tire service life, besides the design with tire, makes the quality related, primarily depends on uses, maintains and in the management whether correct reasonable. Under normal circumstances, the use of travel truck tires rated at $6-6.5$ million meters. However, the actual use situation, a lot of car tires greatly exceeds the quota, to reach 100,000 km and even 200000km; while a considerable number of automobile tires only with 2000-3000km, or less traveling schedules discard. Thus it can be seen, the tire service life has is very big "elasticity". How to prolong the tire service life, people often only pay attention maintain the tire normal pressures, prevents the tire excess load, drives the automobile correctly, prompt maintenance, curing tire and reasonable installment tire, but has neglected effect of the motor car chassis part technical position on the tire wear.
\end{abstract}

Keywords—component; formatting; style; styling; insert (key words)

\section{INTRODUCTION}

The tire is the intermedium of vehicles and path in the vehicles travel process, its running condition can directly reflect that the running condition of vehicles, strength of all functions on vehicles affect completely on the tire, we when defining the tire and use tire hope its pattern even contacts with the road surface, uniform wear in tire use process. However in the design or use process of vehicles, because the part localization expires, will cause the tire localization not to be inaccurate, enabling the tire good even with the ground contact, causes tire the attrition of bureau minister time, causing the service life of tire to drop ${ }^{[1]}$. Because the unusual attrition of tire causes the travel stability variation of vehicles, the chassis suspension fork, travel and steering system spare part cannot according to the technical specification work of normal hypothesis, the working conditions variation reduce the spare part the service life. Meanwhile, the tire unusual attrition not only causes the tire to discard ahead of time, will also create blowout and other breakdowns, threatens the driving safety of automobile seriously, the economic loss that from this causes is selfevident. To being engaged in personnel of motor vehicle maintenance service, through to the mechanism and vehicles of ride stability and analysis of tire location parameter change tire wear to the tire wear position and phenomenon, and takes the corresponding countermeasure and measure, to preventing the tire wears unusually, the increase of service life, the frugal cost and enhancement operational effectiveness and enhancement service efficiency and maintenance quality had very vital practical significance. In this paper, by drawing on existing theoretical basis and scientific research achievements, mainly point of view from the macro Dynamics Tribology to study the tire wear and non-normal wear and tear. Through the relative theory and method, systematic research and scientific discovery, quality technical condition of vehicle chassis parts, has a great influence on the service life of the tire. If the car chassis components do not meet the technical requirements, will accelerate the wear of the tire or mechanical damage, the damage to the tire is the largest of the front wheel alignment is not correct, the brake is not separated completely, loose wheel bearing, axle and chassis frame deformation and spring technology condition is not good. Therefore, these parts and parts should strengthen inspection and adjustment, timely troubleshooting, keep good technical condition.

\section{ADJUST THE CHASSIS PARAMETERS IMPROVE TIRE WEAR}

In order to guarantee that the vehicle has good steering characteristics and driving characteristics, car steering wheel (for front wheel) is provided with a camber angle, toe angle, kingpin inclination and caster angle etc.. Because the positioning angle especially camber angle and toe angle exists, bring bigger impact to the automobile tire wear. Practice shows that, the rapid wear, abnormal wear of automobile tires, tire, except with their quality of wheel dynamic balancing, tire pressure and other factors, another important reason is the camber angle, toe angle and unreasonable matching.

\subsection{Right Front Wheel Positioning}

The automobile wheel alignment including dip angle and kingpin camber, toe in, master. The whichever parameter does not meet the technical requirements, will cause the tire 
abnormal wear, especially the former wheel camber and toe incorrectly wear caused by more serious ${ }^{[2]}$.

Camber is to prevent deformation under load may cause the front axle wheel introverted and setting, its role is to keep the front upright in the car rolling, in order to reduce the running resistance and the tire and the ground friction, tire wear uniform. If the front wheel camber is incorrect, that will increase tire wear. Camber is too large, the outer side of the tire shoulder wear; camber is too small, the inner side of the tire shoulder accelerated wear. When the two front wheel camber unequal, will cause the front wheel outward angle side drag, and front wheel swing, thereby increasing the tire wear

The main cause is abnormal camber deformation front axle, kingpin loose wheel bearings and the gap is too big. Therefore, unilateral tires wear faster when found, should be corrected promptly check these parts ${ }^{[3]}$.

The main role of the front toe is to balance the negative consequences caused by camber of the front wheels in order to ensure integrity of the scroll and improve the steering stability and reduce wear phenomenon dragging wheel on the ground. If the front toe value adjusted properly, it will make the front side trim slippery conditions occur, causing abnormal tire wear. The current value of the wheel before the beam is too large, the center line of the front wheels tend to travel in outward rolling slip inevitably result of wear of the tread laterally from the outside to the inside, the outside tread wear serious raised inner groove; front toe value is too small, the opposite situation with the toe value is too large, the inside of the tread wear serious lateral groove raised. Incorrect toe value for the front tire wear common feature is very flat tread wear, wear very quickly. According to the survey, a unit B1 2020 off-road vehicles toe is $26 \mathrm{~mm}$ (standard is $3 \mathrm{~mm}-5 \mathrm{~mm}$ ), the tire only took $2000 \mathrm{~km}$ driving pattern bald ${ }^{[4]}$.

Cause the front wheel toe-value is not the main reason for checking normal adjustment to toe value is not accurate, the front axle and tie rod deformation, and the front wheel bearings and ball straight horizontal rod loosening, it usually should be careful to check these sites and maintenance work $^{[5]}$. Check the value before the beam, applicationspecific measuring, at a predetermined measurement position of the original measurement, then the original front toe predetermined value adjusted by a rope eyes and infer way is not guaranteed before the front beam value to meet the requirements.

The caster and camber in the role is to ensure the vehicle stability is moving in a straight line, and the steering portability, reducing tire wear. If the caster angle and inclination angle is too large, too small, will cause the front wheel to produce swing and vibration, the impact load increases, aggravate tire wear. Main reasons causing the dip and inclination change caster is sinking and the front axle spring deformation. Kingpin inclination does not meet the requirements, need to remove the front axle of specialized measurement and correction, and the kingpin caster angle does not meet the requirements, it can be adjusted by adding wedge pad between the front axle leaf spring and the front axle.

\subsection{The Front Axle and Rear Axle Parallel}

Automobile front, rear axle parallel axis through the front, after the bridge at both ends of the distance (CA 1091 to $4050 \mathrm{~mm}$, BJ 2020 to $2300 \mathrm{~mm}$ ) to measure the. For a new car, the axis, the bridge at both ends of the distance difference should not exceed $5 \mathrm{~mm}^{[6]}$. If the former, after the bridge is not parallel through the large, will travel in cars like the toe in value is too large, too small, caused by piping sliding tire side and accelerate the tire wear.

Automobile front, rear axle is not parallel usually has two types: fixed not parallel and mobility is not parallel. The existence of fixed is not parallel, tire deflection angle is always in the lower side slip, so tread wear and toe in value is not normal, flat shaped wear. There is variability is not parallel, tire deflection angle is left, right changing, easy to cause the front wheel swing head, so the characteristics of tire wear is tread corrugation.

Main causes of automobile front, rear axle is not parallel to the leaf spring jumping or elastic one side of a leaf spring is too low, before, after the bridge deformation or frame deformation, leaf spring bushing and lug of serious wear and tear or loose. Therefore, in the use of vehicles, to these areas to strengthen the inspection and maintenance, fault found to promptly eliminate.

\subsection{Maintains other Chassis Part Technical}

\section{Condition is Good}

There are bad technical condition of some chassis parts will also cause for tire suddenly quickly suddenly slow (not uniform), and generates vibration Britain, accelerate the tire wear; the front wheel steering angle is too large, meet straight rod will scrap tire cornering; carriage, bad fender technical condition, may contact with the tire in the car the tire, scratched, therefore, before driving and traffic should pay attention to check, found that the problem should be promptly removed, prevent to cause early damage of tire"

\subsection{Braking Characteristics}

The technical position of braking system is not good, often causes applies the brake spontaneously or the individual wheel bubble phenomenon makes the tire mix in the trundle frequently is towing to slide, has the serious attrition. For example: When the gap between brake shoe and brake drum too small or the brake shoe returns to the position to be bad, will create spontaneously automatic; If the brake drum loses the circle, probably causes the wheel in some spot bubble, creates tire periodic delaying, causes the tread protector one has severe wear partially ${ }^{[7]}$ 


\subsection{Steering Mechanism Clearance, Wheel Bearing}

Clearance and Rim Swing Amount to Meet the

\section{Technical Requirements}

Steering gear gap is too small, the rotation of the front wheel bearing is difficult, easy to wear, if the gap is too large, easy to make the automobile front wheel swing, accelerate the tire wear. Hub bearing too loose will cause the wheel shimmy and running instability, wave shape caused by tyre wear, serious when can cause the wheel thrown out. Hub bearing too tight will cause the vehicle driving wandering. All wheel bearing too tight, will make the car sliding distance decreased significantly. And the temperature at the hub was significantly increased in the running process, increase tire wear.

Poor technical condition of these parts, not only will affect wheel alignment and can cause abnormal tire wear directly. Steering mechanism and wheel bearings loose, swinging amount exceeds the required standard rotating wheel vehicles (such as CA 1091 automobile wheel cars swing of less than $10 \mathrm{~mm}$ ), will make the wheels roll, resulting in a wave-shaped tire wear.

\section{CONCLUSIONS}

Factors that affect tire wear are more drivers driving in the ordinary course of operations to note summarizes the traffic in front of the bus and regularly rotate the tires. During use of the tire, the tread is gradually consumed, but because of the discontinuity with the tires, the tire slip amount of the road type, and the affected road arch, the automobile front and rear, left and right wheels of different wear rate. Some tire wear heavy, some light wear and tear, or even unilateral appear uneven tire wear. In order to extend the life of the tire, the tire must be regularly transposition. Timely transposition can extend tire life by about $20 \%$. Tire rotation is usually conducted among secondary vehicle maintenance, road camber in larger areas or summer, a larger difference in tire wear may be appropriate to increase the number of transposition. There are two methods to change a tire and cross-circulation method. Cross transposition law applicable to the regular on the arch of larger vehicles traveling the road, while the law applicable to the regular cycle of change in the relatively flat road vehicles. Found the problem to be immediately removed to prevent damage caused by tire early.

\section{REFERENCES}

[1] Shinto K. Estimation Method of Tire Treadwear on a Vehicle.SAE910168.

[2] Koorosh C. A computer Method ofAnalysis for tire Wear.

[3] K. Elissa, "Title of paper if known," unpublishe Zheng.D,prediction of tire tread wear with FEM steady State Rolling Contact Simulation, tire science and technology. TSTCA,1991.3 (31):122-141.

[4] Atsuo SUEOICA, Takahiro RYU. Polygs \}a\}i Wear ofA \}uto \}obile Tire.[J] JSAE,1997: 209-217
[5] Henk Lupker, Numerical Prediction of Car Tyre Wear Phenomena and Comparison of theObtained Results with Full-Scale Experimental Tests[R]. Helmond,Holland: Chassis and Transport Systems Department T'NO Automotive, 2003

[6] V.K.Goel. Analytical predictions of steady state tire characteristics. Vehicle dynamics system. 2004 .3. 261-275. 